\title{
Amadeus
}

International Multidisciplinary Journal IISSN 2525-8281

DOI: 10.14295/aimj.v4i8.105

\section{Predictors of Adherence to Anti-Hypertensive Therapy: Systematic Review and Meta-Analysis}

Raimundo Monteiro da Silva

Netol

Cícero Rafael Lopes da Silva ${ }^{2}$ Teresa Maria Siqueira Nascimento Arrais

Sâmia Macedo Queiroz Mota Castellão Tavares ${ }^{4}$ Maria Elisa Regina Benjamim de Moura ${ }^{5}$

Emanuela Machado Silva

Saraiva,

Helenicy Nogueira Holanda

Veras $^{7}$

Milana Drumond Ramo

Santana ${ }^{8}$

Poliana Moreira de Medeiros

Carvalho
Abstract: Low adherence to antihypertensive therapy is one of the main modifiable risk factors for several other secondary pathologies. Therefore, we aimed to evaluate the predictors that most interfere in the adherence process. This is a systematic review with meta-analysis that followed the Cochrane recommendations, the search for studies occurred between January and February 2020 using the MeSH: "Hypertensive", "Medication Adherence", "World Population" and "Risk Factors". After applying the filters and criteria 31 studies were selected. The review included studies with intended perspectives and the main predictors were: interventions, satisfaction, cost reduction, the factors were also found: diagnostic impact, medical inertia and execution of the prescribed regimes. The research exposed that more work is needed on health promotion by age group and hypertension $(\mathrm{SAH})$ can be detected and controlled early, readjust high costs, ensure well-being and satisfaction in therapy so that the patient can feel comfortable. Personalized recommendations and constructive comments can drive this process, it is necessary to develop actions aimed at greater compliance by reducing the impact of these diseases and promoting greater control Compliance has always been complex by nature. However, relatively small interventions can substantially improve it.

Keywords: Hypertensive; Medication Adherence; World Population; e Risk Factors.

\footnotetext{
${ }^{1}$ Bolsista do PIBIC-CNPq, discente do curso de enfermagem pela faculdade de Juazeiro do Norte - FJN.

${ }^{2}$ Especialista em Enfermagem Dermatológica pelas Faculdades Integradas de Patos, docente da Faculdade de Juazeiro do Norte - FJN.

${ }^{3}$ Doutoranda em Ciências da Saúde pela FMABC, Docente da Faculdade de Juazeiro do Norte - FJN.

${ }^{4}$ Mestrado em Patologia pela universidade Federal do Ceará, Docente da Faculdade de Juazeiro do Norte - FJN.

${ }^{5}$ Especialização em Urgência e Emergência e Cuidados Intensivos e docente da Faculdade de Juazeiro do Norte FJN.

${ }^{6}$ Mestre em Ciências da Saúde pela Faculdade de Medicina do ABC, Docente da Faculdade de Juazeiro do Norte FJN.

${ }^{7}$ Mestrado em Bioprospecção Molecular pela Universidade Regional do Cariri, Docente da Faculdade de Juazeiro do Norte - FJN.

${ }^{8}$ Pós-doutorado em Ciências da Saúde pela Faculdade de Medicina do ABC - Paulista, Docente da Faculdade de Juazeiro do Norte - FJN.

9 Doutorado em Ciências da Saúde pela Faculdade de Medicina do ABC - Paulista, docente da Faculdade de Juazeiro do Norte - FJN. Contacto: polyfarma2004@yahoo.com.br.
} 


\section{Preditores de Adesão a Terapia Anti-Hipertensiva: Revisão Sistemática e Meta-Análise}

\begin{abstract}
Resumo: A baixa adesão à terapia anti-hipertensiva é um dos principais fatores de risco modificáveis para diversas outras patologias secundárias. Diante disso, objetivou-se avaliar os preditores que mais interferem no processo de adesão. Trata-se de uma revisão sistemática com meta-análise que seguiu as recomendações Cochrane, a busca de estudos ocorreram entre os meses de janeiro e fevereiro de 2020 utilizando os MeSH: "Hypertensive", "Medication Adherence", "World Population" e "Risk Factors". Após a aplicação dos filtros e critérios foram selecionados 31 estudos. A revisão englobou estudos com perspectivas destintas e os principais preditores foram: intervenções, satisfação, redução dos custos, também foram encontrados os fatores: impacto de diagnóstico, inércia médica e execução dos regimes prescritos. A pesquisa expôs que precisa-se trabalhar mais na promoção da saúde, por faixa etária e a hipertensão arterial sistêmica (HAS) possa ser detectada e controlada precocemente, reajustar custos elevados, garantir o bem-estar e satisfação na terapia para que o paciente possa sentir-se confortável. Recomendações personalizadas e comentários construtivos podem impulsionar este processo, é necessário o desenvolvimento de ações que visem uma maior adesão reduzindo o impacto dessas doenças e promovendo um maior controle A adesão sempre foi complexa por natureza. No entanto intervenções relativamente pequenas podem substancialmente melhorá-la.
\end{abstract}

Palavras-chave: Hypertensive; Medication Adherence; World Population; e Risk Factors.

\section{Introdução}

A hipertensão arterial sistêmica (HAS) é uma patologia cardiovascular de etiologia multifatorial e diversas vezes idiopática. A baixa adesão medicamentosa à terapia anti-hipertensiva é um dos principais fatores de risco modificáveis para outras patologias secundárias. Está presente em aproximadamente $69 \%$ dos casos de infarto agudo do miocárdio (IAM) e 77\% dos acidentes vasculares encefálicos (AVE). O controle da pressão arterial permanece insatisfatório em todos os países do mundo, com menos de $50 \%$ dos pacientes hipertensos tratados atingindo as metas terapêuticas recomendadas ${ }^{1-3}$. No Brasil a HAS é um problema comum e sua prevalência varia em torno de $23,6 \%{ }^{1-3}$.

A não adesão aumenta o risco de lesões em órgãos-alvo, tais como AVE, edema pulmonar agudo, síndromes isquêmicas miocárdicas e dissecação aórtica aguda, eleva a vulnerabilidade materna/fetal e mortalidade total. No mundo todo a taxa de mortalidade anual gira em torno de 7,5 milhões de pessoas, é uma condição que possui uma 
indeterminada durabilidade temporal nas fases iniciais e geralmente é assintomática. Por este motivo, muitos pacientes não possuem o hábito de monitorar rotineiramente os valores pressóricos arteriais e identificar o progresso patológico de maneira precoce $^{2,4,37}$.

Diversos fatores podem levar ao desenvolvimento e agravos da HAS, suspeita-se que estes, incluindo entender a importância da adesão ao tratamento medicamento podem estar ausentes no aporte teórico populacional. Com facilidade é possível perceber que muitas vezes assuntos relacionados à HAS como fisiopatologia e tratamento são transmitidos culturalmente de forma errônea e sem embasamento científico. Talvez por isso o controle da pressão arterial (PA) permaneça insatisfatório em todos os países do mundo 5 .

Por meio da análise dos crescentes casos de HAS pode-se inferir um déficit no processo de adesão. Dessa maneira, colocamos a seguinte pergunta: Quais os preditores do processo de adesão à medicação anti-hipertensiva mostram-se mais impulsionantes na população mundial?

O estudo foi desenvolvido na perspectiva de analisar fatores que influenciam na adesão à terapia anti-hipertensiva, contribuindo assim para uma maior compreensão desta temática e propiciar planejamentos de ações voltadas a promoção da saúde global.

Em consideração aos aspectos mencionados, objetivamos avaliar os fatores preditores que proporcionam adesão à farmacoterapia anti-hipertensiva.

\section{Método}

Trata-se de uma revisão sistemática com meta-analiseseguindo as recomendações Cochrane $^{32}$, delineada com base na produção científica de estudos veiculados em periódicos indexados nas bibliotecas digitais National Library of Medicine (Medline), Literatura LatinoAmericana e do Caribe (LILACS), BDENF- Enfermagem e Coleciona-SUS.

Ademais, a revisão seguiu as recomendações metodológicas do protocolo PRISMA (Preferred Reporting Items for Systematic Reviews and Meta-Analyses). ${ }^{34}$ 


\section{Estratégia de Busca}

A questão da pesquisa foi elaborada utilizando a estratégia Population Intervention Comparison Outcome (PICO), isto é, População (P): pacientes com HAS; intervenção (I): Avaliar adesão à medicação anti-hipertensiva; Controle (C): população mundial; "Ouctumes" $(\mathrm{O})$ : identificar preditores de adesão à medicação. Dessa maneira a pergunta norteadora foi: Quais os preditores do processo de adesão à medicação antihipertensiva mostram-se mais impulsionantes na população mundial ${ }^{33}$.

As buscas dos estudos foram realizadas entre os dias 3 de janeiro e 28 de fevereiro de 2020, utilizando um MeSH em cada categoria: P - "Hypertensive” (1),I “Medication Adherence” (2), C - "World Population” (3), O - "Risk Factors" (4).

Os descritores foram inseridos nas bases com o auxílio dos operadores booleanos “AND" e "OR" da seguinte maneira: 2 AND (3 OR 1) AND 4.

\section{Critérios de Elegibilidade do Estudo}

Após a seleção dos artigos que faziam referência aos termos, foi realizado a triagem dos estudos por meio da aplicação dos critérios de busca nas bases de dados, utilizando os seguintes filtros: a) estudos realizados nos últimos 5 anos (2015-2020); b) assunto principal "adesão à medicação"; c) aspecto clínico "terapia"; d) tipo de documento "artigo".

Para composição da amostra foram elencados artigos que: (1) versassem sobre preditores de adesão à medicamentos de maneira específica, (2) publicados nos últimos cinco anos, independentemente do idioma, (3) artigos originais, (4) estudos coorte e caso controle, (5) estudos transversais, (6) ensaios clínicos randomizados (ECR).

Após essa etapa, iniciou-se o processo de translocação dos estudos identificados para uma planilha matriz, estes eram identificados por numeração, base de dados, autor e ano, título, jornal, amostra, principais achados, conclusão e motivo de exclusão, sendo esse processo o marco do começo da etapa de elegibilidade. Nesta operacionalização os estudos estavam sujeitos a serem excluídos se caso não fizessem referência real ao tema proposto da revisão, sendo avaliados pelo título e resumo. Posteriormente, os estudos que não possuíam identificação para serem excluídos foram lidos na íntegra. 
Foram excluídos: I) revisões sistemáticas ou integrativas II) estudos que fugiram do tema III) estudos inconclusivos.

Em função de erros esporádicos dos filtros automáticos dos bancos de dados outros estudos foram exclusos por critérios temporais de publicação.

Os estudos eleitos foram lidos na íntegra e analisados de maneira quantitativa para possibilitar aos pesquisadores uma visão mais ampla da literatura selecionada. Na discordância de inclusão de determinado artigo, um outro revisor era consultado para uma decisão final.

Os estudos que passaram por todo esse processo de revisão e tiveram a decisão final de inclusão, foram atribuídos a uma tabela com as informações subsequentes extraídas: Autor e ano, Objetivos, Tipo de Estudo, $\mathrm{N}^{\mathrm{o}}$ de participantes, principais achados e Risco de Viés em devida ordem que foram encontradas nas bases de dados.

\section{Análise de Dados}

Por fim, os manuscritos foram analisados por meio do pacote estatístico RStudio ${ }^{35} \mathrm{e}$ analisado o risco de viés através da escala de Downs e Black ${ }^{36}$.

\section{Resultados}

A identificação dos trabalhos por meio da busca nas bases de dados, Medline, Lilacs BDENF-enfermagem e Coleciona-Sus resultaram em 15.659 artigos que faziam referência aos termos mencionados. Em sequência, a partir de uma triagem, 83 estudos respeitaram aos critérios de elegibilidade. Após a leitura em sua totalidade foram analisados os quais melhor dariam embasamento para esta pesquisa. Dessa maneira, 23 artigos (3.543.815 participantes) foram inclusos na amostra final do fluxograma PRISMA (figura 1). Os principais preditores foram: custos, ausência de intervenções, idade, conhecimento, impacto do diagnostico, satisfação. Foram estudos realizados em diversas partes do mundo. 
Figura 1. Diagrama de fluxo do processo de elegibilidade dos artigos relacionados a adesão medicamentosa
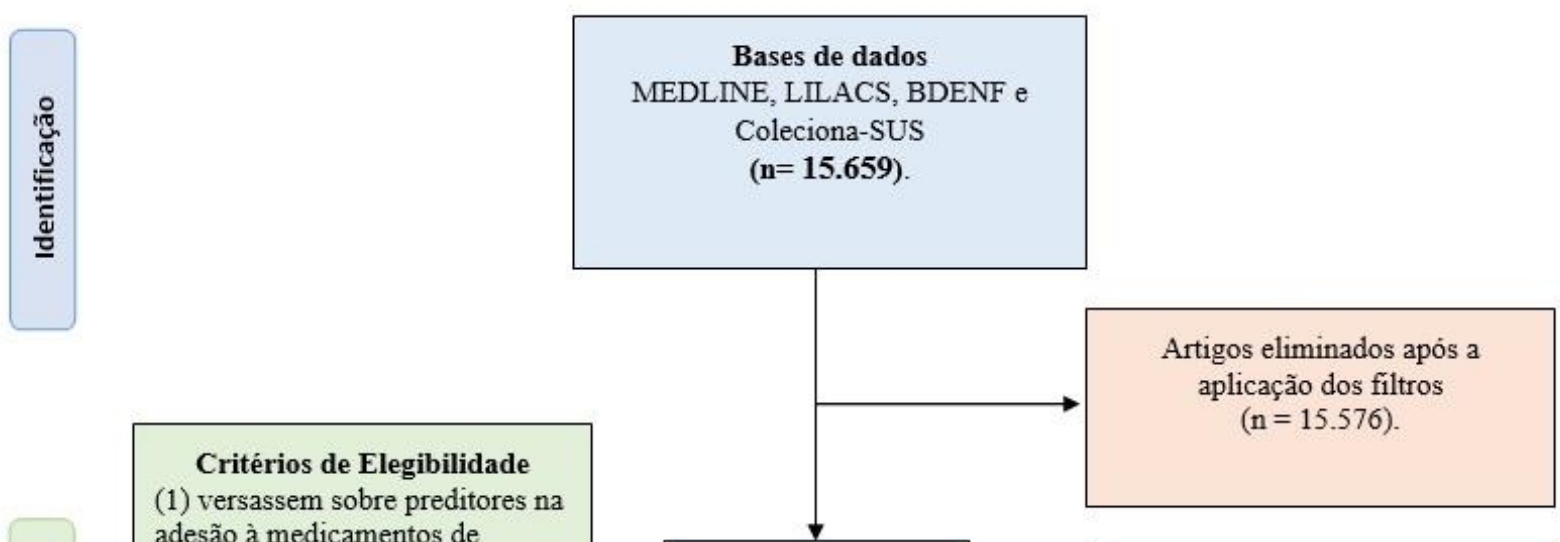
(1) versassem sobre preditores na adesão à medicamentos de maneira específica (2) Pubicados nos ultimos cinco anos, independentemente do idioma

(3) artigos originais

(4) coorte e caso controle

(5) estudos transversais

(6) ensaios clinicos randomizados (ECR)
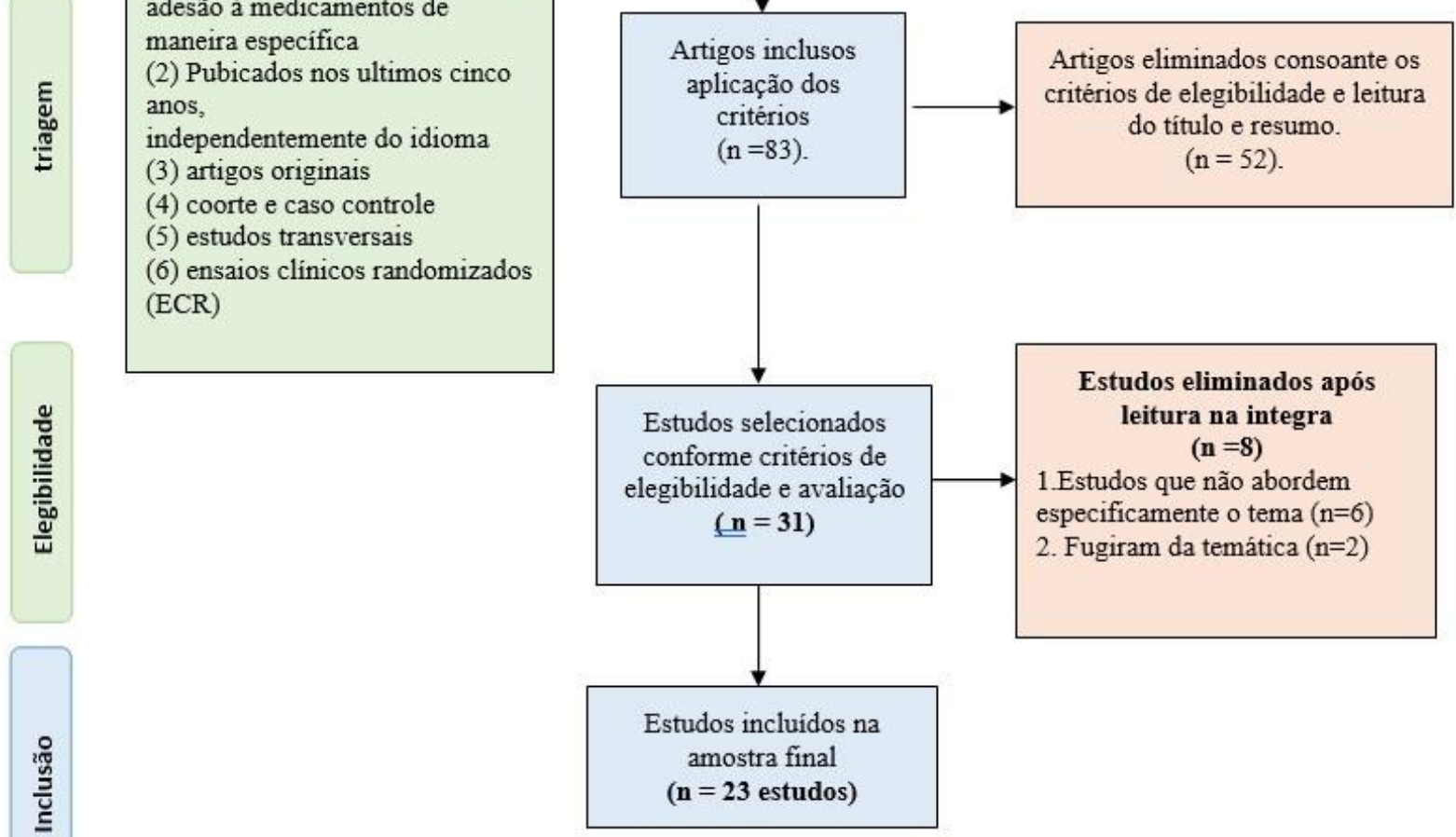

Fonte: Dados da Pesquisa, 2020. 
Tabela 1. Disposição dos artigos segundo autor, ano, objetivo, tipo de estudo, amostra, principais achados e Downs \& Black

\begin{tabular}{clcccc}
$\begin{array}{c}\text { Autor } \\
\text { /Ano } \\
\text { /país }\end{array}$ & objetivo & Tipo de estudo & Participantes & Principais achados & $\begin{array}{c}\text { Downs } \\
\text { Black }\end{array}$ \\
\hline $\begin{array}{l}\text { Baker-Goering/ } \\
\text { 2019/EUA }\end{array}$ & $\begin{array}{l}\text { Identificar a } \\
\text { relação da } \\
\text { adesão anti- } \\
\text { hipertensiva e } \\
\text { custos }\end{array}$ & $\begin{array}{c}\text { Transversal } \\
\text { Retrospectivo }\end{array}$ & $\begin{array}{c}2.897 .548 \\
\text { pacientes }\end{array}$ & $\begin{array}{l}\text { Cerca de } 90 \% \text { dos pacientes } \\
\text { possuíam custos altos e } 30 \% \\
\text { pagou os custos totais de seus } \\
\text { medicamentos }\end{array}$ & 25 \\
& & & $\begin{array}{l}\text { 41\% (N = 1.428.298) daqueles } \\
\text { que receberam uma prescrição } \\
\text { não aderiram à sua medicação. }\end{array}$ \\
\hline
\end{tabular}

\begin{tabular}{|c|c|c|c|c|c|}
\hline $\begin{array}{l}\text { Esther/ } \\
\text { 2019/EUA }\end{array}$ & $\begin{array}{l}\text { Determinara } \\
\text { associação entre } \\
\text { a adesão à } \\
\text { medicação e } \\
\text { satisfação com o } \\
\text { tratamento } \\
\end{array}$ & $\begin{array}{c}\text { Transversal } \\
\text { descritivo }\end{array}$ & $\begin{array}{c}500 \\
\text { participantes }\end{array}$ & $\begin{array}{l}\text { A alta adesão foi } 5 \% \text {, baixa } \\
5,8 \% \text { e moderada } 89,2 \% \text { (446). } \\
\text { Houve uma associação positiva } \\
\text { entre satisfação e adesão. }\end{array}$ & 26 \\
\hline $\begin{array}{l}\text { Jager/ } \\
\text { 2018/EUA }\end{array}$ & $\begin{array}{l}\text { Avaliar a adesão } \\
\text { utilizando } \\
\text { espectrometria } \\
\text { de massa e } \\
\text { cromatografia de } \\
\text { líquido }\end{array}$ & $\begin{array}{l}\text { Open-label } \\
\text { controlado } \\
\text { randomizado }\end{array}$ & $\begin{array}{c}139 \\
\text { Pacientes }\end{array}$ & $\begin{array}{l}\text { Dezesseis pacientes foram } \\
\text { completamente não aderentes } \\
(16 \%), 51 \text { fracamente aderentes } \\
(52 \%) \text { e } 31 \text { aderentes }(32 \%) \text {. } \\
\text {. satisfação e adesão estavam } \\
\text { associadas. }\end{array}$ & 27 \\
\hline $\begin{array}{l}\text { Ashish/ } \\
\text { 2018/ INDIA }\end{array}$ & $\begin{array}{l}\text { Avaliar a adesão } \\
\text { à medicação em } \\
\text { pacientes que já } \\
\text { realizaram } \\
\text { dissecção aórtica } \\
\text { aguda (DAA) }\end{array}$ & Transversal & $\begin{array}{c}74 \\
\text { Pacientes }\end{array}$ & $\begin{array}{c}\text { A adesão foi de } 38 \% \text { a } \\
\text { pontuação Morisky foi } \geq 1.0 \\
\text { para } 27 \text { e } 0 \text { para } 47 \text { pacientes. A } \\
\text { não adesão foi associada com o } \\
\text { aumento da recorrência de dor } \\
\text { torácica. }\end{array}$ & 26 \\
\hline $\begin{array}{l}\text { Adeoye/ } \\
\text { 2019/ Nigéria }\end{array}$ & $\begin{array}{l}\text { Avaliar a adesão } \\
\text { à medicação em } \\
\text { pacientes } \\
\text { nigerianos } \\
\text { hipertensos } \\
\end{array}$ & Coorte & $\begin{array}{c}148 \\
\text { pacientes }\end{array}$ & $\begin{array}{c}\text { Apenas } 4,1 \% \text { tiveram alta } \\
\text { aderência, enquanto que } 68,9 \% \\
\text { tinham aderência moderada, e } \\
27 \% \text { de baixa aderência. }\end{array}$ & 26 \\
\hline $\begin{array}{l}\text { Hennein/ } \\
\text { 2017/EUA }\end{array}$ & $\begin{array}{l}\text { Examinar os } \\
\text { fatores de risco } \\
\text { para a adesão à } \\
\text { medicação }\end{array}$ & Transversal & $\begin{array}{c}1.559 \\
\text { participantes }\end{array}$ & $\begin{array}{l}\text { O risco de baixa adesão foi em } \\
45 \% \text { e no geral } 12 \% \\
\text { apresentaram baixa adesão e foi } \\
\text { associada a uma maior pressão } \\
\text { média arterial diastólica. }\end{array}$ & 26 \\
\hline $\begin{array}{l}\text { Smith/ } \\
\text { 2017/EUA }\end{array}$ & $\begin{array}{l}\text { Avaliar a } \\
\text { viabilidade de } \\
\text { uma intervenção } \\
\text { sobre a adesão à } \\
\text { medicação }\end{array}$ & $\begin{array}{l}\text { Ensaio clinico } \\
\text { randomizado e } \\
\text { controlado }\end{array}$ & $\begin{array}{c}79 \\
\text { pacientes }\end{array}$ & $\begin{array}{l}\text { Demonstrou a viabilidade e } \\
\text { eficácia para atribuir } \\
\text { medicamentos anti- } \\
\text { hipertensivos uma vez por dia } \\
\text { uma vez por dia foi de } 91 \% \text { de } \\
\text { manhã e } 95 \% \text { a noite. }\end{array}$ & 26 \\
\hline $\begin{array}{l}\text { Lauffenburger/ } \\
\text { 2017/EUA }\end{array}$ & $\begin{array}{l}\text { Examinar a } \\
\text { persistência e } \\
\text { adesão à }\end{array}$ & Coorte & $\begin{array}{c}484,493 \\
\text { pacientes }\end{array}$ & $\begin{array}{l}59,4 \% \text { dos pacientes que } \\
\text { estavam persistentes nas } \\
\text { combinações de dose fixa } \\
\text { continuam a usar a mesma } \\
\end{array}$ & 27 \\
\hline
\end{tabular}




\begin{tabular}{|c|c|c|c|c|c|}
\hline & $\begin{array}{l}\text { medicação anti- } \\
\text { hipertensiva }\end{array}$ & & & combinação & \\
\hline $\begin{array}{l}\text { Berhe/ } \\
\text { 2017/Holanda }\end{array}$ & $\begin{array}{c}\text { Avaliar o } \\
\text { impacto de } \\
\text { eventos adversos } \\
\text { a medicamentos } \\
\text { (EAM) e } \\
\text { satisfação }\end{array}$ & Transversal & $\begin{array}{c}925 \\
\text { pacientes }\end{array}$ & $\begin{array}{l}42 \% \text { dos participantes tiveram } \\
\text { baixa, média foi de } 37 \% \text {, e } 21 \% \\
\text { de alta aderência. Satisfação } \\
\text { com o tratamento foi baixa. A } \\
\text { pontuação para satisfação } \\
\text { global foi de } 51(14 \%),\end{array}$ & 26 \\
\hline $\begin{array}{l}\text { Nashilongo/ } \\
\text { 2017/Namíbia }\end{array}$ & $\begin{array}{c}\text { Validar a escala } \\
\text { de conformidade } \\
\text { Hill-óssea e } \\
\text { determinar o } \\
\text { nível e } \\
\text { preditores da } \\
\text { adesão ao } \\
\text { tratamento }\end{array}$ & Transversal & $\begin{array}{c}120 \\
\text { pacientes }\end{array}$ & $\begin{array}{l}\text { O nível de aderência média foi } \\
\text { de } 76,7 \pm 8,1 \% \text {. Nenhum dos } \\
\text { pacientes tinha aderência } \\
\text { perfeita, e menos de metade dos } \\
\text { pacientes } 42,5 \%(50 / 120) \\
\text { tinham níveis aceitáveis de } \\
\text { aderência. A maioria dos } \\
\text { inquiridos eram do sexo } \\
\text { feminino, atingido em educação } \\
\text { menos nível primário }\end{array}$ & 26 \\
\hline $\begin{array}{l}\text { Choi/ } \\
\text { 2017/Coreia do } \\
\text { Sul }\end{array}$ & $\begin{array}{l}\text { Avaliar a } \\
\text { persistência e } \\
\text { aderência com } \\
\text { agentes anti- } \\
\text { hipertensivos. }\end{array}$ & $\begin{array}{c}\text { Coorte } \\
\text { Retrospectivo }\end{array}$ & $\begin{array}{c}20.067 \\
\text { pacientes }\end{array}$ & $\begin{array}{c}\text { As taxas de persistência no } \\
\text { tratamento e adesão foram mais } \\
\text { baixas em pessoas muito idosas } \\
59,5 \% \text { e } 62,8 \% \text {, } \\
\text { respectivamente. E mais } \\
\text { elevadas em pacientes idosos } \\
65,2 \% \text { e } 67,9 \% \text {. }\end{array}$ & 26 \\
\hline $\begin{array}{l}\text { Sung/ } \\
\text { 2017/Taiwan }\end{array}$ & $\begin{array}{c}\text { Examinar os } \\
\text { fatores de risco } \\
\text { para não adesão } \\
\text { em pacientes que } \\
\text { tiveram } \\
\text { acidentes } \\
\text { vasculares } \\
\text { encefálicos } \\
\text { (AVE) }\end{array}$ & Transversal & $\begin{array}{c}9.722 \\
\text { pacientes }\end{array}$ & $\begin{array}{c}\text { A proporção de não aderência } \\
\text { medicação foi de } 71,5 \% \text { entre } \\
\text { os pacientes com HAS que } \\
\text { tiveram AVE. }\end{array}$ & 26 \\
\hline $\begin{array}{l}\text { andrews/ } \\
\text { 2017/EUA }\end{array}$ & $\begin{array}{l}\text { Avaliar e } \\
\text { comparar a } \\
\text { aderência aos } \\
\text { medicamentos } \\
\text { entre adultos }\end{array}$ & $\begin{array}{c}\text { Quase- } \\
\text { experimental }\end{array}$ & $\begin{array}{c}190 \\
\text { pacientes }\end{array}$ & $\begin{array}{l}\text { Um programa com base na } \\
\text { sincronização de identificação } \\
\text { de pacientes em farmácias } \\
\text { comunitárias resultou na } \\
\text { melhoria da aderência. }\end{array}$ & 26 \\
\hline $\begin{array}{l}\text { Daken/ } \\
\text { 2017/ Jordânia }\end{array}$ & $\begin{array}{l}\text { Identificar os } \\
\text { preditores mais } \\
\text { fortes de taxa de } \\
\text { adesão dos } \\
\text { pacientes }\end{array}$ & Transversal & $\begin{array}{c}192 \\
\text { pacientes }\end{array}$ & $\begin{array}{l}\text { A pontuação total media para a } \\
\text { adesão foi } 85,05 \% \\
\text { Maior conhecimento previu } \\
\text { uma boa adesão, visita regular } \\
\text { ao médico. }\end{array}$ & 27 \\
\hline $\begin{array}{l}\text { Bandi/ } \\
\text { 2017/ EUA }\end{array}$ & $\begin{array}{l}\text { Avaliar a } \\
\text { prevalência e os } \\
\text { preditores da } \\
\text { adesão à } \\
\text { medicação anti- }\end{array}$ & Transversal & $\begin{array}{c}1.043 \\
\text { pacientes }\end{array}$ & $\begin{array}{c}\text { Em idosos o conhecimento foi } \\
\text { um preditor positivo e } \\
\text { relacionado com a aderência } \\
\text { A alta aderência foi } \\
\text { significativamente menor em } \\
\text { mais jovens } 24,5 \% \text { vs } 34,0 \% \text {. }\end{array}$ & 27 \\
\hline
\end{tabular}




\begin{tabular}{|c|c|c|c|c|c|}
\hline & hipertensiva & & & & \\
\hline $\begin{array}{l}\text { Park/ } \\
\text { 2016/ Flórida }\end{array}$ & $\begin{array}{c}\text { Determinar o } \\
\text { impacto de } \\
\text { intervenção de } \\
\text { um programa de } \\
\text { telefone } \\
\text { chamada de } \\
\text { lembrete } \\
\end{array}$ & Coorte & $\begin{array}{c}1.126 \\
\text { pacientes }\end{array}$ & $\begin{array}{l}\text { A adesão foi significativamente } \\
\text { maior no grupo de intervenção } \\
\text { PDC (58\% vs } 29 \%) \text {. }\end{array}$ & 27 \\
\hline $\begin{array}{l}\text { Filho/ } \\
\text { 2017/ Coréia } \\
\text { do Sul }\end{array}$ & $\begin{array}{c}\text { Examinar o } \\
\text { papel mediador } \\
\text { da auto-eficácia } \\
\text { na relação entre } \\
\text { a depressão e a } \\
\text { adesão à } \\
\text { medicação } \\
\end{array}$ & Transversal & $\begin{array}{c}255 \\
\text { pacientes }\end{array}$ & $\begin{array}{c}\text { A depressão foi } \\
\text { significativamente } \\
\text { correlacionada negativamente } \\
\text { com a não adesão. }\end{array}$ & 25 \\
\hline $\begin{array}{l}\text { Jeong/ } \\
\text { 2017/ Coréia } \\
\text { do Sul }\end{array}$ & $\begin{array}{l}\text { Avaliar a adesão } \\
\text { à medicação } \\
\text { anti-hipertensiva } \\
\text { em pacientes que } \\
\text { foram } \\
\text { recentemente } \\
\text { diagnosticados } \\
\text { com hipertensão } \\
\text { na Coréia }\end{array}$ & Transversal & $\begin{array}{c}65.919 \\
\text { pacientes }\end{array}$ & $\begin{array}{l}\text { Dos sujeitos do estudo que } \\
\text { visitaram uma instituição } \\
\text { médica, a taxa de prescrição de } \\
\text { anti-hipertensivo foi de } 89,1 \% \text {. }\end{array}$ & 26 \\
\hline $\begin{array}{l}\text { Yang/ } \\
\text { 2016/ EUA }\end{array}$ & $\begin{array}{c}\text { Avaliar o } \\
\text { impacto da } \\
\text { medicação anti- } \\
\text { hipertensiva } \\
\text { (AHM) sobre a } \\
\text { incidência e os } \\
\text { custos }\end{array}$ & Estudo de Coorte & $\begin{array}{c}59.037 \\
\text { pacientes }\end{array}$ & $\begin{array}{c}\mathrm{O} \text { aumento dos custos foi } \\
\text { diretamente relacionado com a } \\
\text { baixa adesão. E no geral } 24,6 \% \\
(\mathrm{n}=14575) \text { tinha uma baixa } \\
\text { adesão. }\end{array}$ & 25 \\
\hline $\begin{array}{l}\text { Kronish/ } \\
\text { 2016/ EUA }\end{array}$ & $\begin{array}{l}\text { Determinar o } \\
\text { efeito de } \\
\text { compartilhament } \\
\text { o de dados de } \\
\text { aderência } \\
\text { eletronicamente } \\
\text { medidos com os } \\
\text { médicos sobre a } \\
\text { gestão da } \\
\text { hipertensão não } \\
\text { controlada. }\end{array}$ & $\begin{array}{l}\text { Ensaio clínico } \\
\text { randomizado por } \\
\text { clusters }\end{array}$ & $\begin{array}{c}100 \\
\text { pacientes }\end{array}$ & $\begin{array}{l}\text { A } \% \text { de visitas foi maior no } \\
\text { grupo de intervenção do que o } \\
\text { grupo de controle ( } 45 \text { de } 65 ; \\
69 \% \text { ) em comparação com (12 } \\
\text { de } 35 ; 34 \% ; p=0,001) \text {. } \\
\text { Os desfechos secundários } \\
\text { incluíram qualidade avaliado } \\
\text { pelo paciente de cuidados e de } \\
\text { comunicação durante a visita. }\end{array}$ & 27 \\
\hline $\begin{array}{l}\text { Garza/ 2015/ } \\
\text { EUA }\end{array}$ & $\begin{array}{c}\text { Medir a eficácia } \\
\text { relativa de } 2 \\
\text { estruturas de } \\
\text { incentivos } \\
\text { econômicos à } \\
\text { base } \\
\text { comportamentais } \\
\text { para melhorar a } \\
\text { adesão à } \\
\text { medicação }\end{array}$ & $\begin{array}{l}\text { Ensaio clínico } \\
\text { randomizado } \\
\text { controlado }\end{array}$ & $\begin{array}{c}36 \\
\text { pacientes }\end{array}$ & $\begin{array}{l}\text { Não houve diferenças } \\
\text { estatisticamente significativas } \\
\text { entre os grupos }(\mathrm{P}>0,05) \text {. } \\
\text { Incentivos financeiros não } \\
\text { mostraram resultados } \\
\text { positivos neste estudo. }\end{array}$ & 26 \\
\hline
\end{tabular}

Filho/

017/ Coréia

o Sul
Avaliar a adesão

anti-hipertensiva

foram

recentemente

diagnosticados

hipertensão

na Coréia
1.126

PDC (58\% vs 29\%).
- visitaram uma instituição médica, a taxa de prescrição de anti-hipertensivo foi de $89,1 \%$.
$\mathrm{O}$ aumento dos custos foi diretamente relacionado com a baixa adesão. E no geral 24,6\% adesão.
A $\%$ de visitas foi maior no grupo de intervenção do que o grupo de controle (45 de 65; \%) em comparação com (12 de $35 ; 34 \% ; p=0,001)$. Os desfechos secundários ncluíram qualidade avaliado pelo paciente de cuidados e de comunicação durante a visita. 


\begin{tabular}{|c|c|c|c|c|c|}
\hline 2015/ China & $\begin{array}{c}\text { fatores } \\
\text { significativamen } \\
\text { te associados } \\
\text { com a adesão à } \\
\text { medicação, } \\
\text { incluindo a } \\
\text { satisfação }\end{array}$ & & & $\begin{array}{l}\text { entrevistados }(55,9 \%) \\
\text { reconheceu algum grau de não } \\
\text { aderência medicação } \\
\text { relacionada a Idade avançada, } \\
\text { viver sozinho e percepção } \\
\text { relacionados com o controlo da } \\
\text { pressão. } \\
\text { Apenas os } 86 \text { participantes } \\
(44,1 \%) \text { foram considerados } \\
\text { como aderentes. }\end{array}$ & \\
\hline $\begin{array}{l}\text { Shireman/ } \\
\text { 2016/ EUA }\end{array}$ & $\begin{array}{l}\text { Avaliar a relação } \\
\text { custo-eficácia } \\
\text { dos } 6 \text { meses } \\
\text { Education Team } \\
\text { e adesão } \\
\text { Monitoramento } \\
\text { (TEAM) de } \\
\text { intervenção para } \\
\text { pacientes }\end{array}$ & $\begin{array}{l}\text { Cluster ensaio } \\
\text { clínico } \\
\text { randomizado }\end{array}$ & $\begin{array}{c}1.250 \\
\text { pacientes }\end{array}$ & $\begin{array}{l}\text { O custo inicial foi de US } \$ 168 \\
\text { por farmácia, baixar custo pode } \\
\text { melhorar o controle da } \\
\text { hipertensão. }\end{array}$ & 27 \\
\hline
\end{tabular}

Fonte: dados da Pesquisa, 2020.

Figura 2: Meta-análise das ações que proporcionam adesão à terapia anti-hipertensiva

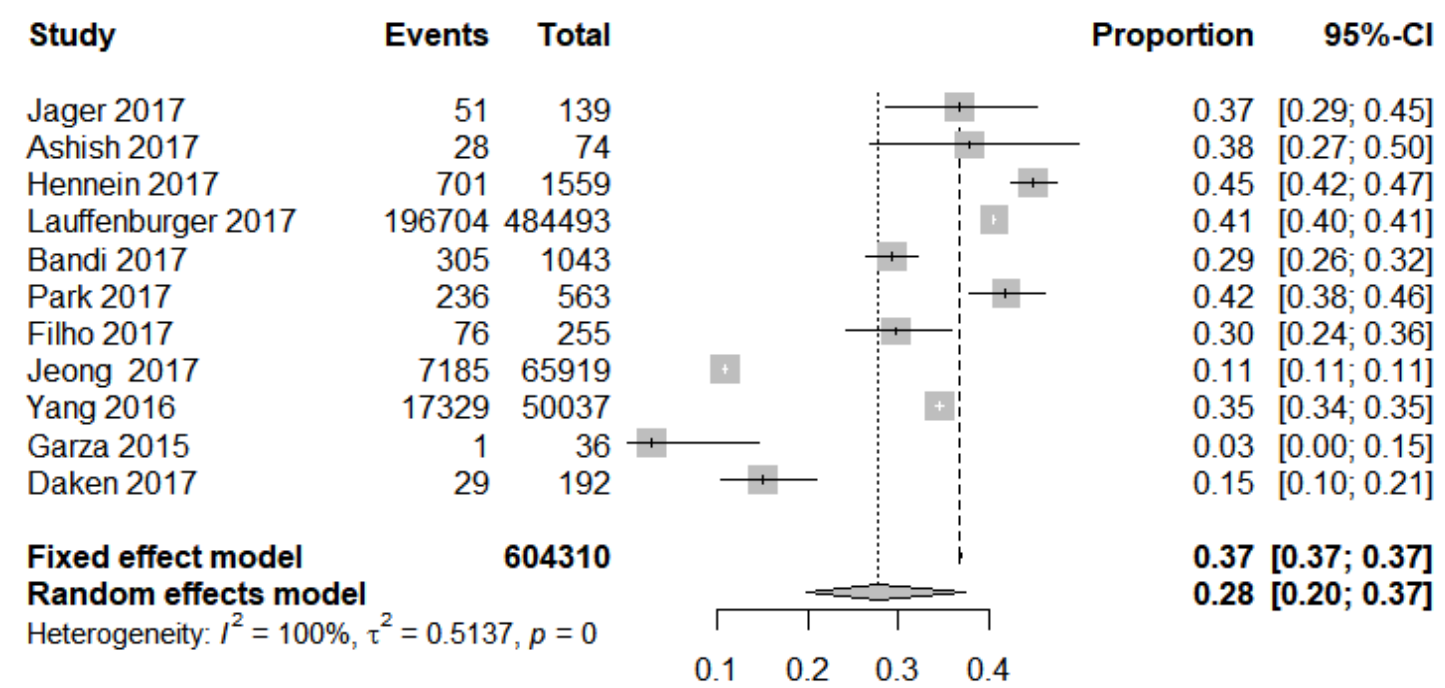

Fonte: Dados da Pesquisa, 2020.

Nos estudos com algum tipo de intervenção ou acompanhamento a análise do efeito final randomizado foi de 0,28 (IC 95\%; $\mathrm{p}=0,0001$ ) uma pontuação baixa e que favorece a adesão, desse modo as intervenções podem aumentar a adesão a terapia antihipertensiva. 
Figura 3: Meta-análise das ações que não proporcionam adesão à terapia antihipertensiva

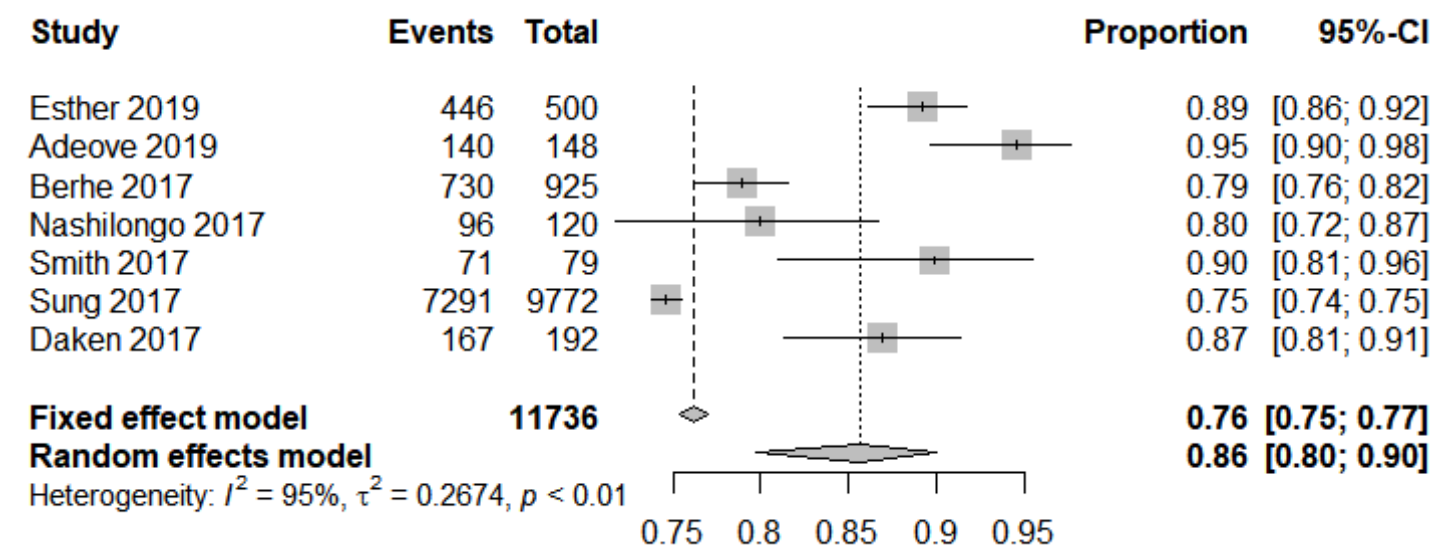

Fonte: Dados da Pesquisa, 2020.

Foi calculado o efeito fixo e randomizado nos estudos heterogêneos, o resultado do efeito resultou em 0,86 (IC 95\%; p <0,01) que representa uma pontuação alta para não adesão.

Houveram diversos preditores de adesão encontrados na literatura, o risco de baixa aderência aumentada em 45\% (IC de 9\%: 25-68\%, P <0,001) associada a uma maior pressão média arterial diastólica $(73 \mathrm{mmHg}$, IC de 95\%: $71-75$ versus $71 \mathrm{mmHg}$, IC95: 70-71; $\mathrm{P}=0,04)^{11}$, fazer dosagens fixas de manhã ou à noite também foi definido em ECR como preditor de boa adesão durante 7 dias a boa adesão foi de $91 \%$ no grupo de manhã e $95 \%$ no braço noite $(\mathrm{p}=0,57)^{12}$.

\section{Idade}

As taxas de persistência no tratamento e adesão foram mais baixas em pessoas muito idosas e mais elevadas em pacientes idosos $65,2 \%$ e $67,9 \%$, respectivamente. $\mathrm{O}$ risco ajustado para o tratamento não foi significativamente maior na persistência dos participantes muito idosos (1,20; IC de 95\%, 1,13-1,27) ${ }^{17}$. Um programa com base na sincronização de identificação de pacientes em farmácias comunitárias resultou na melhoria da aderênciade adultos, essa meta foi atingida por $80,41 \%$ da amostra de 190 
pacientes de um estudo quase experimental. Não convém compara-los pois um não contou com intervenções e o quase experimental, sim. Entretanto isso mostra o quanto intervir pode ser satisfatório ${ }^{19}$.

\section{Intervenção}

Os resultados de um ECR mostraram que $16 \%$ dos pacientes foram completamente não aderentes, os outros $84 \%$ tiveram uma alta e principalmente moderada adesão por meio de espectrometria de massa e cromatografia de líquido (IC de $95 \% 59$ a 78) ${ }^{7}$. Um coorte com 484.493 pacientes revelou participantes iniciantes em combinações de dose fixa, foram $9 \%$ mais propensos a ser persistente na terapia (51,3\% vs $42,1 \%$; risco relativo [RR]: $\mathrm{Cl} 1,09,95 \%: 1,08-1,10)$ e $13 \%$ a mais provável de ser aderente (RR: 1,13 CI 95\%: 1,11-1,14) do que aqueles que começaram em uma única terapia anti-hipertensiva. Pacientes que iniciam combinações de dose fixa tiveram maiores taxas de persistência e aderência ${ }^{14}$.

Um programa de extensão telefônica na adesão à medicação aumentou significativamente a adesão durante o período de pós-intervenção de 17,3\% (33,6; P $<0,001)$ e $13,8 \%(32,3 ; \mathrm{P}<0,001)$ para a intervenção e os grupos controle, respectivamente ${ }^{23}$. Uma intervenção eletrônica também obteve resultados positivos, o número de visitas com gestão clínica apropriada foi maior no grupo de intervenção (comparado ao grupo controle (45 de 65, 69\%; 12 de 35, 34\%; $\mathrm{p}=0,001$ ).

A ausencia de intervenções educativas é um empecilho para a adesão. Em um estudo com 384 pacientes, foram divididos aleatoriamente em 2 grupos utilizando versões do questionário de conhecimento acerca da hipertensão e escala de adesão medicamentosa. O estudo provou que os programas educacionais são úteis para os pacientes no aumento dos níveis de conhecimento sobre hipertensão. Observou-se melhoriana adesão medicamentosa e menores valores pressóricos sistólicos e diastólicos $(131,81 \pm 10,98 \mathrm{mmHg} ; 83,75 \pm 6,21 \mathrm{mmHg})^{9,12,23}$. 


\section{Satisfação}

Dois estudos abordaram de maneira bem específica a correlação da adesão com os índices de satisfação, foram associados com a realização de maior pontuação de aderência $\left(\mathrm{OR}_{1}=21,01 \quad[95 \% \mathrm{Cl}: 1,00 ; 1,01]\right)^{15}$. Os domínios de satisfação com o tratamento estavam associados com a adesão à medicação, e foi estatisticamente significativa $(P=0,000)^{6}$. Não envolver o paciente na tomada de decisões e acima de tudo culpá-los pela má adesão é outro preditor negativo, as recomendações personalizadas e comentários construtivos auxiliam no processo de adesão ${ }^{3}$.

Em um estudo realizado na África onde grande parte não conhecia a importância dos anti-hipertensivos apresentaram desanimo na terapia. A alta adesão foi encontrada em $4,1 \%$ dos participantes, enquanto $68,9 \%$ e $27 \%$ apresentaram adesão moderada e baixa, respectivamente. Além de não conhecer a importância da medicação a baixa motivação para a adesão interfere de maneira significante e o conhecimento foi um preditor independente da adesão à medicação ${ }^{10,22}$.

Tabela 2. Redução dos custos e incentivos econômicos: Não adesão (NA), Adesão à medicação (AM).

Custos

$\mathrm{RR}=0,50$
Redução dos custos

Incentivos econômicos

\begin{tabular}{c|c} 
\%NA & \%AM \\
\hline $49 a$ & $51 b$ \\
\hline $97 \mathrm{c}$ & $3 \mathrm{~d}$ \\
\hline
\end{tabular}

Um estudo intervencional de baixo custo para melhorar o controle da hipertensão e adesão revelou que a quantidade de prescrições anti-hipertensivas pelo grupo de intervenção de custos reduzidos durante o período de 6 meses foi em média 9,9 (SD 1/4 6,2) em comparação com grupo controle 7,9 (SD 1/4 5.6) para o (P 1/40,001) ${ }^{31}$. Em outro randomizado semelhante, ao invés de reduzir custos realizou incentivos econômicos para os hipertensos, o resultado foi ausência de diferença estatística significativa, na adesão entre os grupos de estudo durante esta fase, todos os três grupos tiveram uma baixa adesão $(\mathrm{F}=0,425, \mathrm{P}=0,657)$, diferentemente da redução dos custos, realizar incentivos econômicos não apresenta-se como um preditor tão eficaz na adesão à terapia anti-hipertensiva ${ }^{29}$. 
A probabilidade de não adesão aumentou com os custos elevados (odds ratio ajustados variou: $1,04-1,78 ; \mathrm{P} 0,001)^{4}$. Em um coorte o total de 24,6\% ( $\left.\mathrm{n}=14.575\right)$ tinha uma baixa adesão, a maior aderência foi associada com taxas de eventos cardiovasculares mais baixos em todos os subtipos de eventos de doenças cardiovasculares (DCV) ${ }^{27}$.

Pacientes que usaram medicamentos de marca em diversos estudos experimentais e quase-experimentais encontraram uma relação causal entre reduzir os custos e aumentar a adesão. Outros preditores também encontradosforam: falta de persistência, inércia médica, seguida de uma péssima execução diária dos regimes prescritos. Hoje uma nova abordagem está sendo utilizada que consiste em medir os níveis de medicamentos na urina ${ }^{4,5,27,29,31}$.

\section{Conclusão}

Foram analisados os principais preditores no processo de adesão e pesquisa expôs que precisa-se trabalhar mais na promoção da saúde por faixa etária e a hipertensão arterial sistêmica (HAS) possa ser detectada e controlada precocemente, reajustar custos elevados, garantir o bem-estar e satisfação na terapia para que o paciente possa sentir-se confortável. Recomendações personalizadas e comentários construtivos podem impulsionar este processo, é necessário o desenvolvimento de ações que visem uma maior adesão reduzindo o impacto dessas doenças e promovendo um maior controle. O processo de adesão sempre foi complexo por natureza. No entanto intervenções relativamente pequenas podem substancialmente melhorá-la.

\section{Referências}

1. Aronow, Wilbert S. Optimal Blood Pressure Goals in Patients With Hypertension at High Risk for Cardiovascular Events. Am $J$ Ther | MEDLINE ID: mdl2359102423(1): e218-23, Jan-Feb. 2017.. [Acesso 3 de janeiro de 2020]. Disponível em: <10.1097/MJT.0b013e31827c5372.>.

2. Mariampillai, Julian E; Eskås, Per Anders; Heimark, Sondre; Larstorp, Anne Cecilie K; Fadl Elmula, Fadl Elmula M; Høieggen, Apparent treatment-resistant hypertension - patient-physician relationship and ethical issues. Aud; Nortvedt, Per. 
Jun. Artigo em Inglês | MEDLINE | ID: mdl-28078909. Blood Press; 26(3): 133$138,2017$.

3. Islam MS Hypertension: From Basic Research to Clinical Practice. Islam, Md Shahidul. Adv Exp Med Biol; 956: 1-2, 2017. Disponível em: $<10.1007 / 5584 \_2017 \_30>$

4. Madeleine M. Baker-Goering, PhD 1; Kakoli Roy, PhD 1; David H. Howard, PhD 2 Relação entre adesão ao regime de medicação anti-hipertensiva e custos diretos entre pessoas de 35 a 64 anos com seguro de saúde patrocinado pelo empregador. Prev Chronic Dis 2019.

5. Burnier, M. Drug adherence in hypertension. Pharmacol Res; 125(Pt B): 142-149, 2017 Nov.

6. Oluwole, Esther O; Osibogun, Olatokunbo; Adegoke, Oluseyi; Adejimi, Adebola A; Adewole, Ajoke M; Osibogun, Akin. Medication adherence and patient satisfaction among hypertensive patients attending outpatient clinic in Lagos University Teaching Hospital, Nigeria.

Niger Postgrad Med J; 26(2): 129-137, Apr-Jun. 2019.

7. Jager Sympathy trial. de, Rosa L; van Maarseveen, Erik M; Bots, Michiel L; Blankestijn, Peter J. Br J Clin Pharmacol; Medication adherence in patients with apparent resistant hypertension: findings from the 84(1): 18-24, Jan 2018.

8. Chaddha, Ashish; Erickson, Steven; Kline-Rogers, Eva; Montgomery, Daniel; Woznicki, Elise; Jabara, Justin; Eagle, KimMedication adherence patterns in aortic dissection survivors.. Indian J Med Res; 147(2): 183-188, Feb 2018.

9. Amer, Muhammad; Rahman, Nisarur; Nazir, Saeedur Rashid; Raza, Atif; Riaz, Humayun; Sultana, Misbah; Sadeeqa, Saleha. Impact of pharmacist's intervention on disease related knowledge, medication adherence, HRQoL and control of blood pressure among hypertensive patients Pak J Pharm Sci; 31 (6 (Supplementary): 2607-2616, Nov 2018.

10. Adeoye, Abiodun Moshood; Adebiyi, Akindele Olupelumi; Adebayo, Oladimeji Muritala; Owolabi, Mayowa Ojo. Medication adherence and 24-h blood pressure in apparently uncontrolled hypertensive Nigerian patients. Niger Postgrad Med J; 26(1): 18-24, Jan-Mar. 2019.

11. Rachel Hennein, Shih-Jen Hwang, Rhoda Au. Barreiras à medicação aderência e Links para Cardiovascular Disease Control Risk Factor: O Framingham Heart Study. Volume48, Issue4April 2018 Pages 414-421Internal Medicine Journal. 30 November 2017[acesso 3 de janeiro de 2020]. Disponível em: <https://doi.org/10.1111/imj.13687>.

12. Julia R Smith, Lisa Hillman \&Paul E Drawz. Pharmacist-based antihypertensive medication review and assignment of morning versus evening dosing of once-daily antihypertensive medications: A pilot study to assess feasibility and efficacy in chronic kidney disease patients. Pages 569-573 | Published online: 06 Dec 2017. 
[Acesso

03/01/2020].

Disponível

em:

<https://www.tandfonline.com/doi/full/10.1080/10641963.2017.1411493>.

13. Morawski K,Roya G, Alexis Krumme MS,Julianne Mc DonoughBSErin Durfee BSbLeslieOley MSbNamita Mohta MDaJessie Juusola, Niteesh K.Choudhry. Rationale and design of the Medication adherence Improvement Support App For Engagement-Blood Pressure (MedISAFE-BP) trial American Heart Journal. Volume 186, April 2017, Pages 40-47. [Acesso 4 de janeiro de 2020]. Disponível em: https://doi.org/10.1016/j.ahj.2016.11.007.

14. Lauffenburger, J.C., Landon, J.E. \& Fischer, M.A. J GEN INTERN MED (2017) 32: 619. https://doi.org/10.1007/s11606-016-3972-zEffect of Combination Therapy on Adherence Among US Patients Initiating Therapy for Hypertension: a Cohort Study. J Gen Intern Med; 32(6): 619-625, 2017 Jun. [Acesso 4 de janeiro de 2020]. Disponível em: https://link.springer.com/article/10.1007\%2Fs11606-016-3972-z.

15. Berhe DF, Taxis K, Haaijer-Ruskamp, Flora M. Impact of adverse drug events and treatment satisfaction on patient adherence with antihypertensive medication - a study in ambulatory patients. Volume83, Issue9, September 2017, Pages 21072117. Br J Clin Pharmacol; 83(9): 2107-2117, 2017 Sep. [Acesso 6 de janeiro de 2020]. Disponível em: https://doi.org/10.1111/bcp.13312.

16. Nashilongo, MM, Singu, B., Kalemeera, F. et al. Avaliação da adesão à terapia anti-hipertensiva na atenção primária à saúde na Namíbia: resultados e implicações. Cardiovascular Drugs and Therapy December 2017, Volume 31, Issue 5-6, pp 565-578 [Acesso 6 de janeiro de 2020]. Disponível em: 10.1007 / s10557-0176756-8.

17. Choi, K.H., Yu, Y.M., Ah, Y. et al. Persistence with antihypertensives in uncomplicated treatment-naïve very elderly patients: a nationwide population-based study. BMC Cardiovasc Disord17, 232 (2017). 578 [Acesso 6 de janeiro de 2020]. Disponível em: https://doi.org/10.1186/s12872-017-0665-4.

18. Sung FS. Previously undiagnosed risk factors and medication nonadherence are prevalent in young adults with first-ever stroke. Volume26, Issue12. December 2017. Pages 1458-1464. Disponível em: https://doi.org/10.1002/pds.4250.

19. Andrews $\mathrm{AB}$. The impact of an appointment-based medication synchronization programme on chronic medication adherence in an adult community pharmacy population. Volume42, Issue4. August 2017. Pages 461-466. Disponível em: https://doi.org/10.1111/jcpt.12533.

20. Nidal F. Eshah. (2018) Investigating cardiovascular patients' preferences and expectations regarding the use of social media in health education. Contemporary Nurse 54:1, pages 52-63. Disponível em: https://doi.org/10.1080/10641963.2016.1247164.

21. Tang, K.L., Quan, H. \& Rabi, D.M. Measuring medication adherence in patients with incident hypertension: a retrospective cohort study. BMC Health Serv Res17, 135 (2017). Disponível em:https://doi.org/10.1186/s12913-017-2073-y. 
22. Bandi P. Age-Related Differences in Antihypertensive Medication Adherence in Hispanics: A Cross-Sectional Community-Based Survey in New York City, 20112012. ORIGINAL RESEARCH - Volume 14 - July 13, 2017. Disponível em: https://www.cdc.gov/pcd/issues/2017/16_0512.htm.

23. Park H., Adeyemi A., Wang W., Roane T.E. Impact of a telephonic outreach program on medication adherence in Medicare Advantage Prescription Drug (MAPD) plan beneficiaries. (2017) Journal of the American Pharmacists Association, 57 (1), pp. 62-66.e 2. Disponível em: https://doi.org/10.1016/j.japh.2016.07.006.

24. Son YJ. Depression and medication adherence among older Korean patients with hypertension: Mediating role of self-efficacy. Volume 23, Issue3 June 2017 e12525. Disponível em:https://doi.org/10.1111/ijn.12525.

25. Jeong, H., Kim, H., Lee, K. et al. Medical visits, antihypertensive prescriptions and medication adherence among newly diagnosed hypertensive patients in Korea. Environ Health Prev Med22, 10 (2017). Disponível em: https://doi.org/10.1186/s12199-017-0619-6.

26. Abel W, Greer D. Spiritual/Religious Beliefs \& Medication Adherence in Black Women with Hypertension. Journal of Christian Nursing. 34(3):164-169, /September 2017. Disponível em: JULY/SEPTEMBER 2017.DOI:10.1097/CNJ.0000000000000333.

27. Zhuo Yang, David H. Howard, Julie Will., Fleetwood Loustalot Associação da adesão aos medicamentos anti-hipertensivos com uso em cuidados de saúde e gastos com medicamentos para eventos cardiovasculares agudos. Volume 00, Número 00, " 2016. Disponível em: 10.1097/MLR.0000000000000515.

28. Kronish, I.M., Moise, N., McGinn An Electronic Adherence Measurement Intervention to Reduce Clinical Inertia in the Treatment of Uncontrolled Hypertension: The MATCH Cluster Randomized Clinical Trial. Journal of General Internal Medicine. November 2016, Volume 31, Issue 11, pp 1294-1300. Disponível em: https://doi.org/10.1007/s11606-016-3757-4.

29. Garza KB, Owensby JK. Pilot Study to Test the Effectiveness of Different Financial Incentives to Improve Medication. Adherence. Anais de Farmacoterapia. Vol 50, Issue 1, 2016. Disponível em: https://doi.org/10.1177/1060028015609354.

30. Lo SH, Chau JP, Woo J, Thompson D, Choi K. Adherence to Antihypertensive Medication in Older Adults With Hypertension. Journal of Cardiovascular Nursing. Vol. 31, No. 4, pp 296Y303. August 2016. Disponível em: 10.1097/JCN.0000000000000251.

31. Shiremam T. I, Svarstad BL, Cost-effectiveness of Wisconsin TEAM model for improving adherence and hypertension control in black patients. Jurnal of the American Pharmacists Association Volume 56, Issue 4, Pages 389-396. August, 2016. Disponível em: https://doi.org/10.1016/j.japh.2016.03.002. 
32. Higgins JPT, Verde S, editores. Handook Cochrane para revisões sistemáticas de intervenções: Versão 5.1.0 [atualizado março de 2011. Londres: Cochrane Formação; 2011 [citado 201406 de novembro]. Disponível em: http://handbook.cochrane.org/

33. Santos Cristina Mamédio da Costa, Pimenta Cibele Andrucioli de Mattos, Nobre Moacyr Roberto Cuce. The PICO strategy for the research question construction and evidence search/ A estratégia PICO para a construção da pergunta de pesquisa e busca de evidências.Rev. Latino-Am. Enfermagem [Internet]. 2007 June [cited $2020 \mathrm{Feb}$ 13]; 15(3): 508-511. Available from: http://www.scielo.br/scielo.php?script=sci_arttext\&pid=S0104$11692007000300023 \& \operatorname{lng}=$ en. http://dx.doi.org/10.1590/S010411692007000300023 .

34. Galvão TF, Pansani TS, Harrad D. Preferred reporting items for systematic reviews and meta-analyses: the PRISMA statement. Epidemiol Serv Saude. 2015;24:33542. http://dx.doi.org/10.5123/S1679-49742015000200017.

35. Villacorta, PJ.; Rabelo, CA.; Pelta, DA.; Verdegay, JL. Fuzzy LP: an R Package for Solving Fuzzy Linear Programming Problems. Departamento de Ciências da Computação e Inteligência Artificial. Espanha, Universidade de Granada. Disponível em: <Disponível em: https://cran.rproject.org/web/packages/FuzzyLP/vignettes/FuzzyLP.pdf $>$.

36. Downs SH BN. The feasibility of creating a checklist forthe assessment of the methodological quality both of randomi-sed and non-randomised studies of health care interventions. J Epidemiol Community Heal. 1998.

37. V Diretrizes Brasileiras de Hipertensão Arterial. Arq. Bras. Cardiol. [Internet]. $2007 \mathrm{Sep}$ [cited 2020 Feb 21] ; 89( 3 ): e24-e79. Available from: http://www.scielo.br/scielo.php?script=sci_arttext\&pid=S0066782X200700150001 2\&lng=en. https://doi.org/10.1590/S0066782X2007001500012.

\section{How to cite this article (APA format):}

Silva Neto, Raimundo Monteiro da; Silva, Cícero Rafael Lopes da; Arrais, Teresa Maria Siqueira Nascimento; Tavares, Sâmia Macedo Queiroz Mota Castellão; Moura, Maria Elisa Regina Benjamim de; Saraiva, Emanuela Machado Silva; Veras, Helenicy Nogueira Holanda; Santana, Milana Drumond Ramos; Carvalho, Poliana Moreira de Medeiros.(2020). Predictors of Adherence to Anti-Hypertensive Therapy: Systematic Review and Meta-Analysis. Am. In. Mult. J., February to May. (8) 4, 50-67.

Received: 03/07/2020;

Accepted: 03/26/2020. 